\title{
Scaling breakdown: A signature of aging
}

\author{
P. Allegrini, ${ }^{1,2}$ J. Bellazzini, ${ }^{3}$ G. Bramanti, ${ }^{4}$ M. Ignaccolo, ${ }^{2,5}$ P. Grigolini, ${ }^{2,4,6}$ and J. Yang ${ }^{2}$ \\ ${ }^{1}$ Istituto di Linguistica Computazionale del Consiglio Nazionale delle Ricerche, Area della Ricerca di Pisa-S. Cataldo, Via Moruzzi 1, \\ 56124 Ghezzano-Pisa, Italy \\ ${ }^{2}$ Center for Nonlinear Science, University of North Texas, P.O. Box 311427, Denton, Texas, 76203-1427 \\ ${ }^{3}$ Dipartimento di Ingegneria Aerospaziale dell'Università di Pisa, Via Caruso, 56100 Pisa, Italy \\ ${ }^{4}$ Dipartimento di Fisica dell'Università di Pisa and INFM, Via Buonarroti 2, 56126 Pisa, Italy \\ ${ }^{5}$ Center for Nonlinear Science, Texas Woman's University, P.O. Box 425498, Denton, Texas 76204 \\ ${ }^{6}$ Istituto di Biofisica del Consiglio Nazionale delle Ricerche, Area della Ricerca di Pisa-S. Cataldo, Via Moruzzi 1, \\ 56124 Ghezzano-Pisa, Italy
}

(Received 27 November 2001; published 12 July 2002)

\begin{abstract}
We prove that the Lévy walk is characterized by bilinear scaling. This effect mirrors the existence of a form of aging that does not require the adoption of nonstationary conditions.
\end{abstract}

DOI: 10.1103/PhysRevE.66.015101

PACS number(s): 89.75.Da, 05.40.Fb, 05.45.Df, 05.45.Tp

In the last few years Lévy flights and walks have become a popular field of investigation for physicists [1,2]. They are as widely applied in nonlinear, fractal, chaotic, and turbulent systems as Brownian motion is in simpler systems. Their theoretical foundation rests on one hand on the generalized central limit theorem (GCLT) [3], and on the other hand, on the renormalization group (RG) [4]. The RG serves the purpose of explaining the physical origin for fluctuations with diverging second moment, and the GCLT proves that the diffusion process generated by these fluctuations is characterized by probability distribution functions (PDF) that are as stable as the Gaussian distributions generated by fluctuations with finite second moment. The GCLT refers to the case where the fluctuations are uncorrelated and the random walker, at regular intervals of time, makes jumps of arbitrarily large intensity. If the distribution of these jumps lengths is already stable, the resulting diffusion process is christened Lévy flight [1,2]. This physical condition is judged to be unrealistic, and for this reason in the last 17 years Lévy diffusion has been studied under the form of Lévy walk [5-7], where a certain time is needed to complete each jump depending on its length, in this paper being proportional to it. Later research $[2,8,9]$ has established that the Lévy walks are processes with memory. Here we plan to prove a further interesting property: Lévy walk is characterized by aging, reflected by the emergence of bilinear scaling, and aging is, quite surprisingly, compatible with the adoption of stationary conditions.

Let us consider a sequence $\left\{\tau_{i}, s_{i}\right\}$, with $i=0,1, \ldots, \infty$. The numbers $\tau_{i}$ are random numbers with the distribution density

$$
\psi(\tau)=\frac{(\mu-1) T^{\mu-1}}{(T+\tau)^{\mu}} .
$$

Note that we shall set $\mu>2$ so as to ensure the mean time $\langle\tau\rangle$ to exist and be finite: it is easy to prove that $\langle\tau\rangle$ $=T /(\mu-2)$. The numbers $s_{i}$ have the values 1 and -1 , determined by the coin tossing rule. Let us imagine two archetypal individuals, Jerry and Bob, in action to realize with this time series a diffusion process without age and with age, respectively. Both Bob and Jerry create first an infinite trajectory for the diffusion variable $y(l)$, generated by the sequence $\left\{\tau_{i}, s_{i}\right\}$, according to a criterion of their own choice. Note that for any sequence $\left\{\tau_{i}, s_{i}\right\}$ there exists only one trajectory $y(l)$. Then they consider the values that the trajectory $y(t)$ gets at times $L$ and $L+t$ so as to create, for each $L$, a trajectory defined by $x=0$ at $t=0$ and $x(t)=y(T+t)$ $-y(T)$ at $t>0$, and thus an infinite number of trajectories from the original single trajectory.

Let us illustrate first the criterion adopted by Jerry to construct the trajectory $y(l)$. The time $l$ of Jerry is discrete, and it corresponds to the number of random drawings done to get the position $y(l)$. Formally

$$
y(l)=W \sum_{i=0}^{l} \tau_{i} s_{i}
$$

with the parameter $W>0$ serving the purpose of arbitrarily scaling jump intensities. Then, Jerry builds up the trajectories $x(t)$, each of them characterized by the label $L$, and determines the PDF at a generic time $t$. After a fast transient, according to the GCLT [3], the Fourier transform of this PDF, $\hat{p}_{L}(k, t)$, becomes

$$
\hat{p}_{L}(k, t)=\exp \left(-|k|^{\mu-1} b t\right)
$$

where

$$
b \equiv W^{\mu-1} T^{\mu-2} \cos \left[\frac{\pi}{2}(\mu-1)\right] \Gamma(3-\mu),
$$

with $\Gamma(\cdot)$ denoting the well known Gamma function. Let us imagine that Jerry keeps secret the values of $W$ and $T$. In this case, it is not possible to establish at which time $t$ Jerry began his experiment, observing the PDF shape. In fact, this observation might lead us to determine $b$, and this quantity depends on three unknowns, $W, T$, and $t$. Furthermore, if Jerry adopted for $\psi(\tau)$ a stable form, Lévy flight, even the fast transition process would be annihilated. 
Let us see how Bob builds up his single generating trajectory. For a generic time $l$, let us consider the time $l_{N}$ fitting the property that $l_{N} \equiv \tau_{0}+\tau_{1}+\tau_{N-1}<l$, while $l_{N}+\tau_{N} \geqslant l$. In this condition, Bob's generating trajectory is given by

$$
y(l)=W\left[\tau_{0} s_{0}+\tau_{1} s_{1}+\cdots+\tau_{N-1} s_{N-1}+\left(l-l_{N}\right) s_{N}\right],
$$

with $W$ playing here the role of velocity intensity. Let us call $i$ th event the random selection of the pair $\left\{\tau_{i}, s_{i}\right\}$. The walker starts moving immediately after the occurrence of the first event and spends the whole time $\tau_{0}$ in a condition of uniform motion, laminar phase [5], before the occurrence of the second event, at which time the motion direction can also be inverted, and so on. In this case, the adoption of infinitely many trajectories $x(t)$ corresponds to creating a stationary condition, with the walkers staying in the first laminar phase with a time distribution corresponding to equilibrium [10]. Before illustrating the crucial result of this paper, based on the stationary condition, let us discuss briefly the nonstationary case [11] when Bob has really at his disposal infinitely many sequences $\left\{\tau_{i}, s_{i}\right\}$, and consequently many trajectories $y(l)$. This would be equivalent to an out-of-equilibrium condition, which would relax to the equilibrium condition with a relaxation prescription $\propto 1 / l^{\mu-1}$ [12]. How many events will have been realized by Bob for any of his walker up to time $l$ ? For $l \gg\langle\tau\rangle$, the number of events, $N$, is expected to be $N$ $=l /\langle\tau\rangle$. Actually, we can set all this on a rigorous basis using a theorem by Feller [13]. At any instant of time $l$ the number of random walkers for which $\sum_{i=1}^{N-1} \tau_{i} \leqslant l$ and $\sum_{i=1}^{N} \tau_{i}>l$, is not fixed, and its mean value, $\langle N\rangle$, is given by

$$
\langle N\rangle=\frac{l}{\langle\tau\rangle}\left[1+\frac{T^{\mu-2}}{(3-\mu)} \frac{1}{l^{\mu-2}}\right] .
$$

Note that each event implies a fixed amount of entropy increase, due to the random prescriptions adopted to realize an event. Thus, Eq. (6) shows that the rate of entropy increase is not constant. It is constant either in the exponential case (ordinary statistical mechanics, with $\mu=\infty$ ) or in the asymptotic time limit, namely, in the scaling regime compatible with the perspective of thermodynamic equilibrium. One might be tempted to consider Eq. (6) to reflect a nonstationary condition that in the case $\mu<3$ would live forever.

It is not so. First of all, the relaxation to equilibrium is faster than the memory effect, $1 / l^{\mu-1}$ vs $1 / l^{\mu-2}$. A careful study of the stationary condition confirms this remark. The Lévy walk realized by Bob can be described as the solution of the differential equation $d x / d t=\xi(t)$, where $\xi(t)$ is a stochastic velocity keeping the value $W(-W)$ for a time $\tau_{i}$, with or without a change of sign at the end of this sojourn time, as a result of the coin tossing. In the stationary case the correlation function $\left\langle\xi\left(t_{1}\right) \xi\left(t_{2}\right)\right\rangle /\left\langle\xi^{2}\right\rangle$ depends on $t=\mid t_{1}$ $-t_{2} \mid$, and it is denoted by $\Phi_{\xi}(t)$. Using the renewal theory $[5,14], \Phi_{\xi}(t)$ is related to $\psi(\tau)$ by

$$
\Phi_{\xi}(t)=\frac{1}{\langle\tau\rangle} \int_{t}^{+\infty}\left(t^{\prime}-t\right) \psi\left(t^{\prime}\right) d t^{\prime}=\left(\frac{T}{T+t}\right)^{\mu-2} .
$$

It is remarkable that this correlation function has the same asymptotic properties as the correction term to the condition of constant rate of entropy increase established by Eq. (6).

We expect $p(x, t)$ to become a Lévy stable distribution for $t \rightarrow \infty$, according to the GCLT prediction of Eq. (3), as fully confirmed by numerical simulation (see, for instance, Ref. [15]). However, this transition process is infinitely slow. In fact, we note that at any time $t$ a finite number of Bob's trajectories $x(t)$ are still in the same laminar region where they were at $t=0$. These trajectories are moving by uniform motion with velocity $W$ and $-W$, thus establishing peaks of decreasing intensity and an abrupt truncation of the PDF, at its right and left border, respectively. To evaluate this number, or the probability that a trajectory contributes to the propagation front, $I_{p}(t)$, we must refer ourselves to the probability distribution $\psi_{a s}(\tau)$. This is the alternating signs distribution, or distribution of times through which the trajectory keeps moving in the positive or negative direction, a distribution not coinciding with $\psi(\tau)$, due to the random choice of sign. The two distributions are related to one another through their Laplace transforms, $\hat{\psi}_{a s}(s)$ and $\hat{\psi}(s)$, respectively, by means of [11]

$$
\hat{\psi}_{a s}(s)=\frac{\hat{\psi}(s)}{2-\hat{\psi}(s)} .
$$

Using the renewal theory [5] we prove that

$$
I_{p}(t)=\frac{1}{\langle\tau\rangle_{a s}} \int_{t}^{+\infty}\left(t^{\prime}-t\right) \psi_{a s}\left(t^{\prime}\right) d t^{\prime} .
$$

These trajectories keep moving by ballistic motion and thus contribute to the propagation fronts signaled in the numerical treatment by two ballistic peaks. It becomes thus evident that a very plausible form for the PDF is given by

$$
p(x, t)=K(t) p_{L}(x, t) \theta(W t-|x|)+\frac{1}{2} \delta(|x|-W t) I_{p}(t),
$$

where $\theta(\cdot)$ denotes the Heaviside step function. $p_{L}(x, t)$ is a distribution that for $t \rightarrow \infty$ becomes identical to the antiFourier transform of Eq. (3), and $K(t)$ is a time-dependent factor ensuring the normalization of the distribution $p(x, t)$, thereby taking the form

$$
K(t)=\frac{1-I_{p}(t)}{1-2 \int_{W t}^{+\infty} p_{L}(x, t) d x} .
$$

Using the method of Laplace transform, it is straigthforward to prove that $\lim _{t \rightarrow \infty} K(t)=1$ and that

$$
\lim _{t \rightarrow \infty}\left[I_{p}(t)-\Phi_{\xi}(t)\right]=0 .
$$

On the basis of these arguments we reach the conclusion that in the asymptotic time limit Eq. (10) becomes identical to 


$$
p(x, t)=p_{L}(x, t) \theta(W t-|x|)+\frac{1}{2} \delta(|x|-W t) \Phi_{\xi}(t) .
$$

This equation coincides with an earlier, less rigorous, prediction [14], and allows us to prove that it is possible to determine the age of Lévy walk. We can determine the age of the diffusion experiment created by Bob, even if Bob adopts the stationary condition $[5,11]$ and keeps secret the values of $W$ and $T$. To do so, we measure the distance of one ballistic peak from the other, the diffusion coefficient $b$ of $p_{L}(x, t)$ of Eq. (4) and the intensity of the two ballistic peaks. All these three quantities can be expressed in terms of the unknown quantities $t, W$, and $T$. The distance between the two peaks is $2 W t$, the diffusion coefficient is given by $b$ $=W(T W)^{\mu-2} \sin [\pi(\mu-2) / 2] \Gamma(3-\mu)[15]$, and the peak intensity by $\Phi_{\xi}(t)$.

What about the scaling of Bob's diffusion in the time asymptotic limit? The current techniques of analysis, of which the detrended fluctuation analysis is a popular example (see Ref. [16] for an update), are inadequate to answer this question. In fact, these techniques aim at evaluating scaling through the second moment of PDF: a correct procedure in the Gaussian case that becomes questionable in the nonGaussian case here under study. Here we prove the emergence of the Lévy scaling as a form of dominating scaling by using the technique of diffusion entropy (DE) $[17,18]$. According to Barkai [19] and an earlier work [20], in the time asymptotic limit Bob's diffusion should yield

$$
p(x, t)=\frac{1}{t^{\delta}} F\left(\frac{x}{t^{\delta}}\right)
$$

with $\delta=1 /(\mu-1)$ (Lévy scaling). The DE is nothing but the Shannon entropy of this PDF, defined by

$$
S(t)=-\int_{-\infty}^{+\infty} d x p(x, t) \ln p(x, t)
$$

By plugging Eq. (14) into Eq. (15) we get immediately

$$
S(t)=A+\delta \ln t .
$$

Thus, the representation of the numerical results in a logarithmic time scale is a very simple way to detect scaling. In Fig. 1 we see that, in full accordance with the arguments of Refs. [20,19], in the long-time limit Bob's diffusion fits the Lévy scaling condition.

As pointed out by Barkai [19], the work of other authors $[21,22]$, who discovered the biscaling nature of the Lévy walk, seems to cast doubt on this conclusion, which has been judged by him to be an attractive foundation of the fractional derivative method. To address this issue, In accordance with the methods used to deal with multifractality [23,24], we study theoretically and numerically the fractional moment $\left\langle|x|^{q}\right\rangle$, which is expected to yield

$$
\left\langle|x|^{q}\right\rangle=\int_{-\infty}^{+\infty} p(x, t)|x|^{q} d x \approx t^{\xi_{q}} .
$$

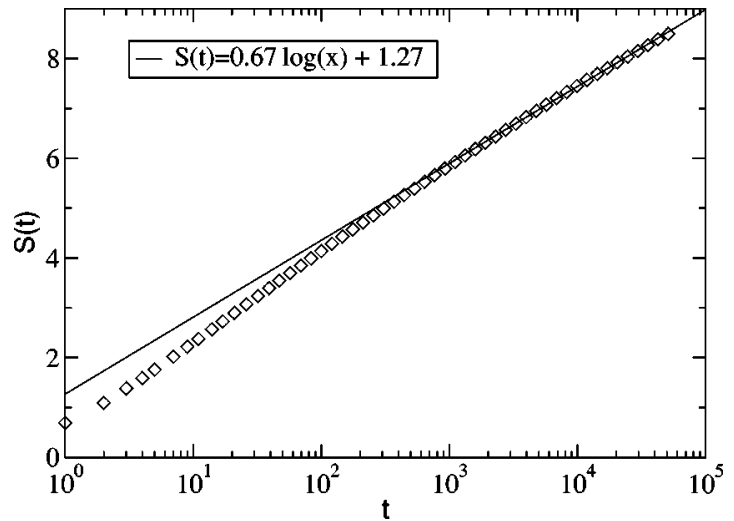

FIG. 1. Diffusion entropy as a function of time $t$. Dots, numerical evaluation of $S(t)$ for Bob's experiment with $\mu=2.5, T \approx 1.0$. Bob's walkers are derived from a single trajectory of total length 25918673 time units (see the text for details). The solid line is a best fit to the numerical results, in the asymptotic regime, by means of the theoretical prediction $S(t)=2 / 3 \ln (t)+$ const, with $\delta=2 / 3$ stemming from the Lévy scaling.

The power index $\xi_{q}$ plays a critical role. If the condition of Eq. (14) applied, with the function $F(\cdot)$ having all moments finite, $\xi_{q}$ as a function of $q$ would be a straight line. According to the theory of Refs. [23,24], this would be an indication of monofractality. In the case under discussion, all PDF moments are finite, thereby making the monofractal condition possible. Using Eq. (13) it is straigthforward to predict that

$$
\xi_{q}=\delta q
$$

for $q<\mu-1$, and

$$
\xi_{q}=q-\mu+2
$$

for $q>\mu-1$. This is so because for $q<\mu-1$ the Lévy distribution, even with no truncation, would produce a finite fractional moment. The prediction for $q>\mu-1$ is dictated by the peak intensity of Eq. (13). With the same kind of

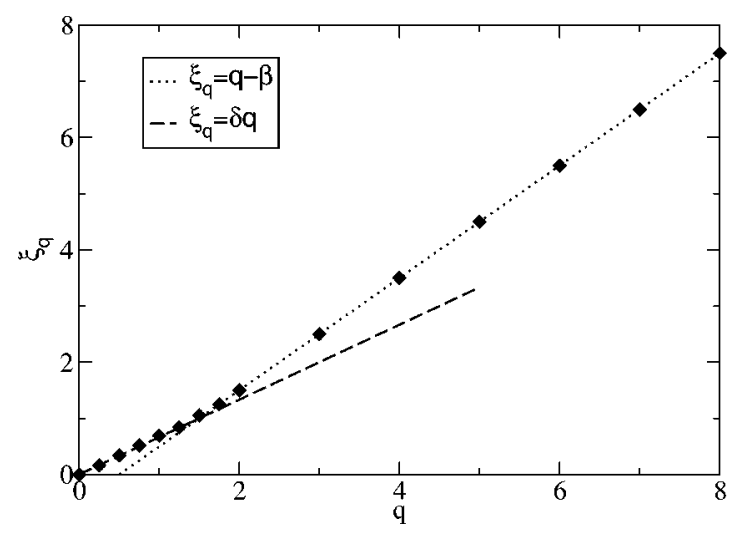

FIG. 2. Multifractal index $\xi_{q}$ as a function of $q$. Dots, numerical evaluation of $\xi_{q}$ for Bob's experiment using the same data as those of Fig. 1. Dashed line, $\xi_{q}=\delta q$, with $\delta=2 / 3$ predicted by the Lévy scaling; dotted line, $\xi_{q}=q-\beta$, with $\beta \equiv \mu-2=0.5$. 
calculation it is possible to derive the same result for $\mu>3$ when the central part of the PDF would be a truncated Gaussian distribution.

These theoretical predictions are very satisfactorily supported by the numerical results, as proved by Fig. 2. The satisfactory agreement between theoretical prediction and numerical experiment yields support to Eq. (13), and to the aging perspective stemming from this key formula as well. If we compare the theoretical predictions of Eqs. (18) and (19) to the calculation done by the authors of Ref. [2] in the case of Lévy flight (Jerry's random walkers), we find agreement for $q<\mu-1$ and disagreement for $q>\mu-1$, where we find a finite value while they find $+\infty$ : aging postpones to an infinitely large time the exact equivalence of Lévy walk with Lévy flight. Note that the key formula of Eq. (13) allows us to generalize the prediction of the work of Refs. [21,22], which would be confined to integer values of $q$.
In the literature of aging, some authors, (see, for instance, Ref. [25]), make the theoretical proposal of assessing aging through the adoption of two-time correlation functions, aging implying a dependence on both times, rather than only on their difference. The adoption of this view here would prevent us from distinguishing the dichotomous case, yielding Lévy statistics, from the case where $\xi(t)$ is a Gaussian noise, with the same correlation function. In this case, it is trivial to prove that scaling is linear, rather than bilinear. The correlation function to use properly is $\Phi_{\xi}(t)$, reflecting the stationary condition of Bob's experiment. In conclusion, we show that the intermittent nature of the process under study yields aging, and our theory makes it possible to determine age, in spite of the stationary condition.

Financial support from ARO, through Grant No. DAAD19-02-0037 is gratefully akcnowledged.
[1] J. Klafter, M.F. Shlesinger, and G. Zumofen, Phys. Today 49(2), 33 (1996).

[2] G. Zumofen, J. Klafter, and M.F. Shlesinger, Lect. Notes Phys. 519, 15 (1998).

[3] B.V. Gnedenko and A. N. Kolmogorov, Limit Distributions for Sums of Independent Random Variables (Addison Wesley, Cambridge, MA, 1954).

[4] N. Goldenfeld, Lectures on Phase Transitions and the Renormalization Group (Perseus Book, Reading, 1992).

[5] T. Geisel, J. Nierwetberg, and A. Zacherl, Phys. Rev. Lett. 54, 616 (1985).

[6] M.F. Shlesinger and J. Klafter, Phys. Rev. Lett. 54, 2551 (1985).

[7] M.F. Shlesinger, B.J. West, and J. Klafter, Phys. Rev. Lett. 58, 1100 (1987).

[8] G. Trefán, E. Floriani, B.J. West, and P. Grigolini, Phys. Rev. E 50, 2564 (2564).

[9] G.M. Viswanathan, V. Afanasyev, S.V. Buldyrev, E.J. Murphy, P.A. Prince, and H.E. Stanley, Nature (London) 381, 413 (1996).

[10] G. Zumofen and J. Klafter, Physica A 196, 102 (1993).

[11] G. Zumofen and J. Klafter, Phys. Rev. E 47, 851 (1993).

[12] M. Ignaccolo, P. Grigolini, and A. Rosa, Phys. Rev. E 64,
026210 (2001).

[13] W. Feller, Trans. Am. Math. Soc. 67, 98 (1949).

[14] P. Allegrini, P. Grigolini, and B.J. West, Phys. Rev. E 54, 4760 (1996).

[15] M. Annunziato and P. Grigolini, Phys. Lett. A 26931 (2000).

[16] K. Hu, P.Ch. Ivanov, Z. Chen, P. Carpena, and H.E. Stanley, Phys. Rev. E 64, 011114 (2001).

[17] N. Scafetta, P. Hamilton, and P. Grigolini, Fractals 9, 193 (2001).

[18] P. Grigolini, L. Palatella, and G. Raffaelli, Fractals 9, 439 (2001).

[19] E. Barkai, Phys. Rev. E 63, 046118 (2001).

[20] G. Zumofen, J. Klafter, and A. Blumen, Chem. Phys. 146, 3081 (1987).

[21] K.H. Andersen, P. Castiglione, A. Mazzino, and A. Vulpiani, Eur. Phys. J. B 18, 447 (2000).

[22] P. Castiglione, A. Mazzino, P. Muratore-Ginnaneschi, and A. Vulpiani, Physica D 134, 75 (1999).

[23] R. Benzi, G. Paladin, G. Parisi, and A. Vulpiani, J. Phys. A 17, 3521 (1984).

[24] G. Paladin and A. Vulpiani, Phys. Rep. 156, 149 (1987).

[25] N. Pottier and A. Mauger, Physica A 282, 77 (2000). 